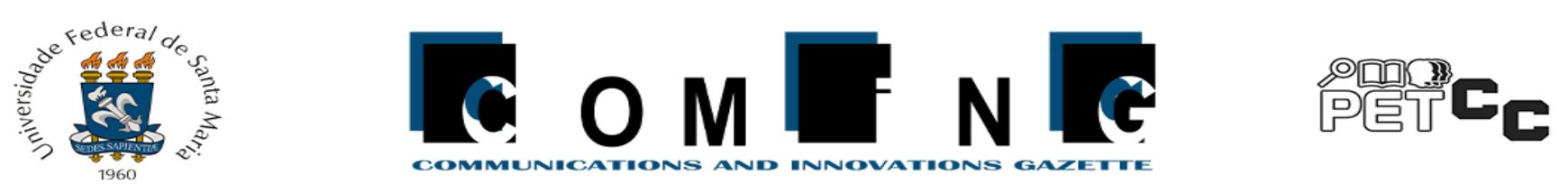

\title{
A\&B Game Jam - Projeto extensionista de estímulo ao desenvolvimento de jogos
}

\author{
Daniel C. C. Boll ${ }^{1}$, Davi M. Giacomel ${ }^{1}$, Felipi L. Matozinho ${ }^{1}$, Gustavo L. Nomelini ${ }^{1}$, \\ Igor F. Negrizoli ${ }^{1}$, Leonardo Vanzin ${ }^{1}$, Lucas G. de A. Amorim ${ }^{1}$, Lucas V. de Sá ${ }^{1}$, \\ Marco A. Damo ${ }^{1}$, Mateus E. R. da Silveira ${ }^{1}$, Mateus K. Camara ${ }^{1}$, Matheus N. \\ Ismael $^{1}$, Roberta A. da S. Alcantara ${ }^{1}$, Marcio S. Oyamada ${ }^{1}$ \\ ${ }^{1}$ Centro de Ciências Exatas e Tecnológicas - Universidade Estadual do Oeste do Paraná \\ (UNIOESTE) \\ Cascavel - PR - Brasil \\ \{daniel.boll,davi.giacomel1,felipi.matozinho,gustavo.nomelini,igor.negrizoli,leonardo.vanzin,lucas.amori \\ m3,lucas.sa1,marcio.oyamada,marco.damo,mateus.silveira,mateus.camara,matheus.ismael,roberta.alcanta \\ ra\}@unioeste.br
}

\begin{abstract}
A\&B Game Jam is a short-time game development marathon that aims to foster the game development scene in the region of Cascavel, Paraná. The focus of $A \& B$ is not on competition, but rather on learning, socialization and fun. Organized since its third edition by the Computer Science PET group from Western Paraná State University, the marathon had its third edition held onsite at the university in 2019 and its fourth edition held online in 2020. The event has already attracted dozens of participants from the external community, stimulating them to develop their first game in a cooperative and learning environment, always being well evaluated by them. This work discusses the idea behind $A \& B$, all the activities related to the organization of the third and fourth editions, as well as their goals and the lessons learned by the organization.
\end{abstract}

Resumo. A A\&B Game Jam consiste em uma maratona de desenvolvimento de jogos em um curto período de tempo e tem por objetivo estimular o cenário de desenvolvimento de jogos na região de Cascavel, Paraná. O foco da A\&B não é a competição, mas sim o aprendizado, a socialização e a diversão. Organizada desde sua terceira edição pelo grupo PET Ciência da Computação da Universidade Estadual do Paraná, a maratona teve sua terceira edição realizada presencialmente na universidade em 2019 e a quarta edição realizada online em 2020. O evento já atraiu dezenas de participantes da comunidade externa, estimulando-os a desenvolver o seu primeiro jogo em um clima de cooperação e aprendizado, sendo sempre bem avaliada por eles. O presente trabalho discute a proposta da $A \& B$ e todas as atividades referentes à organização da terceira e da quarta edições, bem como seus resultados e os aprendizados adquiridos pela organização.

\section{Introdução}

Game Jams são eventos de criação rápida de jogos onde um jogo é criado em um espaço de tempo relativamente curto a partir de limitações pré-definidas e cujos resultados são compartilhados publicamente [Kultima 2015]. Em outras palavras, são maratonas de desenvolvimento de jogos com regras específicas que regem este desenvolvimento. Em geral, elas têm duração de 48 horas, sendo realizadas em um único fim de semana. Entretanto, podem durar vários dias ou apenas algumas horas. 
Muito além do mero desenvolvimento de jogos, Game Jams são eventos onde há a criação e aprimoramento de comunidades, compartilhamento de experiências e transformação de processos, estimulando a inovação e a criatividade. Elas promovem um novo tipo de prática que requer dos participantes raciocínio rápido, flexibilidade e inovação em curtos períodos de desenvolvimento. A colaboração e o companheirismo entre diferentes times trabalhando em um mesmo espaço fomentam um espírito de comunidade em cada evento e a realização de múltiplos eventos permite que tais comunidades cresçam [Locke et al. 2015]. Participantes de Game Jams buscam trabalhar em equipe e se divertir enquanto desenvolvem jogos e têm, nos fatores de aprendizado e sociabilização, suas principais motivações para participar desse tipo de evento [Reng et al. 2013].

Além dos fatores sociais e educacionais mencionados, um grande benefício proporcionado por Game Jams é o fomento à indústria de jogos. Em 2018, ela gerou cerca de US\$137,9 bilhões, um valor superior ao do mercado das indústrias de música e de cinema juntas [LPE 2018]. Apenas nos Estados Unidos, tal indústria gera mais de 65 mil empregos diretos e cerca de 220 mil empregos indiretos [Takahashi 2017].

Nesse contexto, a A\&B Game Jam foi criada em 2018 pelo acadêmico Mateus Karvat Camara e pelo publicitário Javan Bocchi, visando estimular o cenário de desenvolvimento de jogos na cidade de Cascavel, Paraná. Suas duas primeiras edições foram realizadas online, com participantes exclusivamente da cidade de Cascavel, nos meses de julho e dezembro desse mesmo ano. A partir da experiência de seus fundadores com Game Jams e Hackathons, a A\&B foi concebida para ser um evento com foco na colaboração entre os participantes. Assim, ela não se trata de uma competição - como muitas outras Game Jams - e conta com algumas atividades (milestones) que devem ser realizadas pelos participantes no decorrer da maratona, a fim de propiciar maior socialização e troca de conhecimentos entre eles, bem como momentos de diversão e aprendizado.

A partir da terceira edição, a A\&B Game Jam passou a ser organizada e realizada como atividade de extensão pelo grupo PETComp do curso de Ciência da Computação da Universidade Estadual do Paraná (Unioeste), buscando, além dos objetivos supracitados, estimular a participação de membros da comunidade local em atividades relacionadas à computação, bem como divulgar o grupo e suas atividades para a comunidade externa. A terceira edição foi realizada de forma presencial entre os dias 30 de agosto e 01 de setembro de 2019 no campus Cascavel da Unioeste e a quarta edição foi realizada de forma remota entre os dias 02 e 07 de setembro de 2020.

O presente artigo descreve as atividades relacionadas à organização e realização da III e IV A\&B Game Jam. A Seção 2 descreve o processo de preparação de cada edição, enquanto a Seção 3 analisa e compara os dados levantados sobre cada edição.

\section{Organização}

Em ambas as edições da A\&B Game Jam, todos os membros do grupo PETComp auxiliaram na organização e realização do evento, dividindo-se em subgrupos encarregados de tarefas específicas como, por exemplo, divulgação, organização das milestones e inscrições. Visto que a A\&B não é uma competição, todos os membros da organização têm liberdade para também participar da maratona. Apesar disso, o tema é 
definido pelo petiano coordenador do projeto e revelado apenas aos membros da organização que não serão participantes.

A organização dos eventos começa meses antes do período de realização, iniciando com a definição da data, sempre visando a conveniência dos petianos organizadores e o aumento da possibilidade de inscrição de participantes externos à universidade. Após a definição da data, a organização de cada evento se dá mediante atividades específicas para a execução de divulgação, inscrição e definição da programação da Game Jam, como a criação dos posters apresentados nas Figuras 1 e 2.

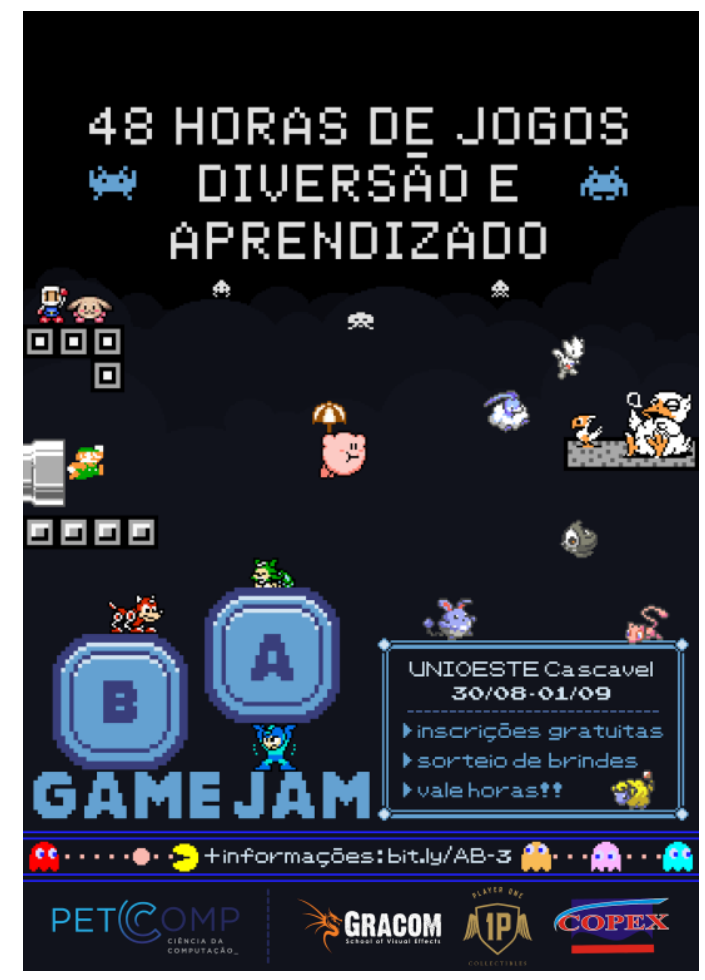

Figura 1 - Pôster de divulgação da III A\&B Game Jam

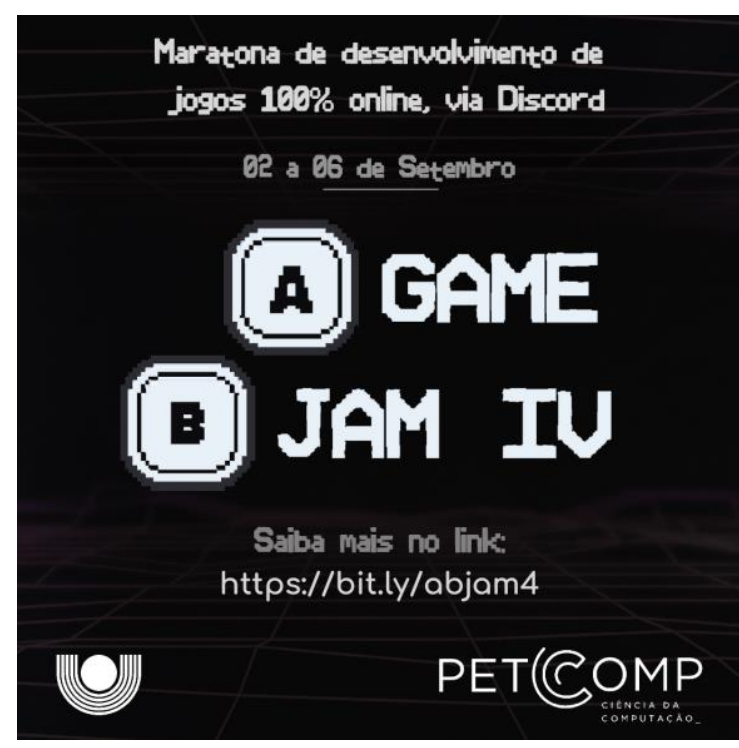

Figura 2 - Pôster de divulgação da IV A\&B Game Jam

Em ambas as edições, foram criadas páginas web na plataforma Game Jolt a fim de centralizar as informações dos eventos [Petcomp 2019] [Petcomp 2020]. Para a realização das inscrições, utilizou-se formulários na plataforma Google Forms, nos quais os interessados em participar dos eventos deveriam inserir informações referentes à sua equipe e ao seu histórico de participação em Game Jams. Todos os inscritos nos formulários foram adicionados a um grupo de WhatsApp para facilitar a comunicação.

\subsection{A\&B Game Jam}

Inicialmente, um pequeno grupo se reuniu para definir quais seriam as milestones desta edição da $A \& B$, que seria a primeira edição presencial do evento. Definiu-se duas atividades: "Conhecendo a Universidade" e "Show me the good stuff".

Para a realização da primeira milestone, foram confeccionados crachás para todos os participantes com um personagem de um jogo e metade de um $Q R$ Code. Cada participante deveria procurar pelo participante com um personagem do mesmo jogo que 
o seu e unir os crachás a fim de revelar o código completo. Tal $Q R$ Code indicaria um local da universidade que deveria ser visitado pela dupla. Lá eles encontrariam outro código com uma pergunta sobre jogos que deveria ser discutida pela dupla e relatada ao coordenador do evento ou outro membro da organização. Esta atividade tinha por objetivo integrar participantes de grupos distintos e apresentar a universidade a membros da comunidade externa.

A segunda milestone, inspirada em eventos de Hackathon, consistia na apresentação dos resultados parciais sobre aspectos do desenvolvimento. Em horários predeterminados para cada quesito, os participantes deveriam mostrar ao coordenador do evento o andamento do seu jogo nos quesitos: conceito, arte e programação. Esta exposição visava auxiliar os participantes no processo de desenvolvimento, contribuindo para uma experiência mais agradável e um aprendizado mais efetivo.

Após a definição das milestones, decidiu-se que elas dariam pontos aos participantes que as completassem, a fim de estimular sua realização. Tais pontos, ao final do evento, seriam utilizados como número para o sorteio de brindes. Tendo isso em vista, o grupo buscou patrocínio com empresas locais para a obtenção de brindes a serem sorteados. Desse modo, o evento pôde ser realizado de forma gratuita.

Nos três finais de semana prévios ao evento, foram realizados minicursos preparatórios com os temas "Criação de Arte para Game Jams", "Programação de Jogos em Unity" e "Game Design". Tais minicursos propiciaram um aprendizado prévio aos participantes com pouca experiência em desenvolvimento de jogos.

Após a criação da página web e a definição dos patrocinadores, o pôster da Figura 1 foi criado e impresso por uma das empresas patrocinadoras, iniciando a etapa de divulgação. Esta foi realizada de modo presencial com visitas a cursos universitários da cidade relacionados às diferentes habilidades necessárias a um grupo em uma Game Jam: arte, programação e música. Além disso, cada membro da organização divulgou o evento em grupos de WhatsApp que poderiam ter pessoas interessadas, em suas redes sociais e para pessoas próximas.

Na semana de realização, solicitou-se aos participantes que se inscrevessem no evento pelo Sistema de Eventos da Unioeste (SGEV), para que fosse possível gerar certificados de participação e para que todos pudessem acessar a rede $W i-F i$ da universidade durante o evento.

O espaço cedido pela universidade para a realização do evento foi de 6 salas de aula, das quais 3 foram reservadas para que os participantes realizassem $o$ desenvolvimento dos jogos, 2 para que eles dormissem e a última foi reservada para o lobby. Este foi organizado para ser um espaço de interação e descontração, contando com decorações temáticas e algumas atividades extras opcionais, como arremesso de bolinha de papel num cesto de lixo. Durante todo o evento, havia ao menos um membro da organização presente no lobby para atender a quaisquer necessidades dos participantes. Na noite do dia 31 de Agosto, o lobby foi utilizado para um momento de integração em que, mediante contribuição voluntária dos participantes interessados, se serviu pizza. $\mathrm{O}$ local também foi utilizado para a realização do encerramento, onde cada equipe mostrou o jogo desenvolvido à equipe de organização e aos demais participantes.

Além disso, na noite do dia 30 de Agosto, foi realizada uma pequena palestra de abertura em um mini auditório da universidade, na qual a proposta do evento, suas 
dinâmicas e regras foram explicadas a todos os participantes. Neste momento, o tema da Game Jam foi revelado aos participantes, anunciando que todos os jogos deveriam ser desenvolvidos com a temática de "Universidade".

\subsection{A\&B Game Jam}

Em virtude da pandemia de Covid-19, a quarta edição da A\&B foi realizada virtualmente. Assim como nas primeiras duas edições do evento, que não foram organizadas pelo grupo PETComp, esta edição foi realizada pela plataforma Discord, pela qual os participantes puderam se comunicar entre si e com a organização.

Seguindo sugestões dos participantes da III A\&B Game Jam, esta edição foi realizada próxima a um feriado, sendo selecionado o de Independência do Brasil. Antes da definição do período de realização do evento, membros da organização entraram em contato com participantes das edições anteriores para verificar a viabilidade de realização desta edição. Com base nas informações coletadas, definiu-se que o evento seria realizado em um prazo maior, com cinco dias para desenvolvimento e um dia para o compartilhamento dos jogos finalizados, a fim de viabilizar a participação de pessoas que no momento estavam trabalhando em regime de home office. Assim, definiu-se que o evento ocorreria entre os dias 02 de Setembro e 07 de Setembro. Sua abertura foi realizada no dia 02, às 19 horas, com uma palestra transmitida via YouTube do Mestrando em Gamificação Igor Arnaldo de Alencar, tratando sobre o tema Mods. Após a palestra, o coordenador do evento explicou aos participantes como ocorreriam as atividades e revelou o tema desta edição, que foi "Peste Bubônica". Os participantes tiveram então até o dia 06 de Setembro, às 23 horas, para desenvolver seu jogo e submetê-lo na plataforma Game Jolt. No dia 07 de Setembro, às 18 horas, foi realizada a apresentação dos jogos desenvolvidos via Discord.

Por se tratar de uma edição online, a expectativa da organização foi que o número de inscritos seria significativamente menor que o número da terceira edição. Tendo isso em vista, optou-se por não buscar patrocínio para a realização do evento com empresas parceiras, uma vez que o ganho de valor para tais empresas seria muito pequeno caso houvesse um número baixo de inscritos.

Outro agravante da pandemia foi que a divulgação não pôde ser realizada presencialmente nas instituições de ensino da região, de modo que o contato com elas se limitou a e-mails enviados aos colegiados dos cursos visitados no ano anterior. Para a divulgação nas redes sociais e WhatsApp, o pôster da Figura 02 foi criado e compartilhado por tais canais.

Tendo em vista a interação limitada de um evento online, as milestones da IV A\&B Game Jam foram pensadas de modo a propiciar maior interação entre os participantes. Todas elas deveriam ser enviadas pelos participantes em canais específicos do servidor do Discord do evento. Tais milestones podem ser classificadas como Desenvolvimento, Integração e Diversão.

Dentre as milestones de Desenvolvimento, de modo similar à III A\&B, os participantes deveriam compartilhar etapas do desenvolvimento do jogo, mostrando aos demais o Conceito do jogo, o Título e Capturas de tela do desenvolvimento.

Nas milestones de Integração, os participantes contavam com um canal de Dicas onde poderiam compartilhar aprendizados adquiridos em edições anteriores ou mesmo 
durante a edição atual a fim de auxiliar participantes menos experientes. Além disso, um canal de Tech Support propiciou que participantes de uma equipe pudessem solicitar auxílio de outras pessoas na resolução de bugs e demais problemas técnicos enfrentados no desenvolvimento. Por fim, na milestone de Foto do Grupo os participantes poderiam conhecer uns aos outros, compartilhando uma foto da equipe no Discord do evento. Devido à pandemia, os participantes foram instruídos a não se reunirem para tirar uma foto, mas a tirar fotos individuais fazendo o símbolo de "A" (referente ao nome do evento) com a mão e posteriormente editá-las digitalmente para criar a do grupo.

As milestones de Diversão, por sua vez, buscavam apenas propiciar uma pausa no desenvolvimento do jogo e um breve momento de descontração e relaxamento. Durante todo o evento, os participantes poderiam compartilhar fotos de comida e enviar memes em um canal específico. Na sexta-feira, dia 04 de Setembro de 2020, uma atividade de integração com o jogo Stop Online foi realizada com participantes e membros da organização. No sábado, dia 05 de Setembro de 2020, durante a tarde, os participantes deveriam mandar fotos de si mesmos emulando a foto do cantor e compositor Ednaldo Pereira [Pereira 2020].

Nesta edição, a inscrição dos participantes no SGEV foi opcional, sendo necessária apenas para aqueles que desejavam obter o certificado de participação do evento.

\section{Resultados}

\subsection{A\&B Game Jam}

O evento contou com 76 inscrições e 50 participantes efetivos, sendo $65 \%$ dos inscritos da comunidade externa. Dos 76 inscritos, algumas estatísticas foram levantadas, estando as mais relevantes presentes nas Tabelas 1 e 2 .

A Tabela 1 permite concluir que a III A\&B Game Jam alcançou seu objetivo de estimular o cenário de desenvolvimento de jogos na região, visto que cerca de $79 \%$ dos inscritos nunca haviam participado de nenhuma Game Jam anteriormente. Dos 16 inscritos que já haviam participado de Game Jams, 10 deles haviam participado exclusivamente de outras edições da A\&B Game Jam, de modo que a porcentagem de inscritos que não participaram de nenhuma Game Jam exceto a A\&B é de $92 \%$.

Tabela 1 - Número de Game Jams já participadas anteriormente à III A\&B

\begin{tabular}{|c|c|c|c|c|c|}
\hline Número de Game Jams & 0 & 1 & 2 & 3 & 4 \\
\hline Total de pessoas & 60 & 10 & 3 & 2 & 1 \\
\hline Participou exclusivamente da A\&B & - & 7 & 3 & - & - \\
\hline
\end{tabular}

A Tabela 2, por sua vez, demonstra o sucesso das diferentes estratégias de divulgação adotadas. Cerca de $26 \%$ das inscrições vieram da divulgação realizada nas universidades da cidade e cerca de $13 \%$ da divulgação por grupos de WhatsApp. Acredita-se que, a partir dessas inscrições, tais pessoas convidaram seus amigos, o que 
resultou num grande número de participantes que descobriram o evento a partir de seus amigos (43\%).

Tabela 2 - Número de inscritos que descobriram o evento por cada meio de divulgação utilizado na III A\&B

\begin{tabular}{|c|c|c|c|c|c|}
\hline \multicolumn{7}{|c|}{ Como descobriu o evento } \\
\hline Amigos & Faculdade & Organização & WhatsApp & Site & Outro \\
\hline 33 & 20 & 11 & 10 & 1 & 1 \\
\hline
\end{tabular}

Os 50 participantes se organizaram de forma espontânea em 9 grupos com diferentes números de membros, tendo o menor grupo 4 integrantes e, o maior, 8 integrantes.

Após o evento, solicitou-se a todos os participantes que respondessem um formulário de avaliação criado na plataforma Google Forms. Nele, foram abordadas questões referentes à eficácia das milestones, do trabalho da equipe de organização do evento, estrutura física e organização dos espaços, dos pontos positivos e negativos do evento, bem como uma questão avaliando o interesse dos participantes em uma Game Jam não gratuita. $\mathrm{O}$ resumo de algumas das questões do formulário é apresentado nas Tabela 3 e 4.

Tabela 3 - Respostas para as perguntas sobre as Milestones e a estrutura física da III A\&B

\begin{tabular}{|c|c|c|c|}
\hline \multicolumn{2}{|c|}{ Tema da pergunta: Milestones } & \multicolumn{2}{c|}{ Tema da pergunta: Estrutura física } \\
\hline $\begin{array}{c}\text { Aspectos comumente } \\
\text { citados e opiniões } \\
\text { recorrentes }\end{array}$ & $\begin{array}{c}\text { Número de } \\
\text { respostas } \\
\text { (percentual das } \\
\text { respostas) }\end{array}$ & $\begin{array}{c}\text { Aspectos comumente } \\
\text { citados e opiniões } \\
\text { recorrentes }\end{array}$ & $\begin{array}{c}\text { Número de respostas } \\
\text { (percentual das } \\
\text { respostas) }\end{array}$ \\
\hline Relaxamento & $8(16 \%)$ & $\begin{array}{c}\text { Positivo (comentário } \\
\text { genérico) }\end{array}$ & $16(32 \%)$ \\
\hline Interação & $13(26 \%)$ & $\begin{array}{c}\text { Internet (reclamação) } \\
\text { Tomadas } \\
\text { (reclamação) }\end{array}$ & $4(22 \%)$ \\
\hline Desenvolvimento & $19(38 \%)$ & $\begin{array}{c}\text { Banheiro com odor } \\
\text { desagradável }\end{array}$ & $5(10 \%)$ \\
\hline Indiferente & $10(20 \%)$ & & 4 \\
\hline Comentários de destaque: & “Acredito que em eventos desse tipo seria chato se não existissem milestones” \\
\hline
\end{tabular}


Tabela 4 - Respostas para a pergunta sobre os pontos negativos e positivos da III A\&B

\begin{tabular}{|c|c|c|c|}
\hline \multicolumn{4}{|c|}{ Tema da pergunta: Pontos positivos e negativos do evento } \\
\hline Pontos positivos & Número de respostas & Pontos negativos & Número de respostas \\
\hline Interação & 12 & Banheiro & 8 \\
\hline Desenvolvimento & 9 & Internet & 8 \\
\hline $\begin{array}{l}\text { Cerimônia de } \\
\text { Encerramento }\end{array}$ & 8 & Colchonetes & 5 \\
\hline Tudo & 7 & Ambiente & 3 \\
\hline \multicolumn{4}{|c|}{ Comentários de destaque: } \\
\hline \multicolumn{4}{|c|}{$\begin{array}{l}\text { "O melhor foi a interação com outras equipes, justamente por ser um evento não competitivo, } \\
\text { todos buscaram se ajudar, trocar conhecimento e ideias além de interagir de forma geral, acho } \\
\text { que se a jam tivesse um intuito competitivo essa interação não iria acontecer do mesmo jeito" }\end{array}$} \\
\hline $\begin{array}{l}\text { "Saber que eu estava } \\
\text { ganhar um prêmio de }\end{array}$ & $\begin{array}{l}\text { ndo o jogo pq eu q } \\
\text { o ambiente muito } \mathrm{m}\end{array}$ & $\begin{array}{l}\text { fazer um jogo, e } \\
\text { eve e divertido" }\end{array}$ & pq estava esperando \\
\hline
\end{tabular}

Tal formulário demonstrou uma opinião extremamente positiva de todos os participantes a respeito do evento, fornecendo algumas sugestões de melhorias para edições posteriores. As críticas focaram em aspectos do espaço de realização do evento e, por conta disso, a organização pretende realizá-lo em outro local nas próximas edições presenciais.

Além disso, em uma reunião interna do PET após a Game Jam, cada um dos membros deu seu feedback para avaliar a atividade. Todos demonstraram satisfação com a condução do evento e indicaram interesse em dar continuidade a ele para o planejamento de atividades a serem realizadas pelo grupo no ano de 2020 .

\subsection{A\&B Game Jam}

Esta edição da $\mathrm{A} \& \mathrm{~B}$ contou, como já previsto pela organização, com um número menor de participantes, totalizando 45 inscritos e 35 participantes efetivos, sendo $69 \%$ das inscrições provenientes da comunidade externa. As Tabelas 5 e 6 apresentam os dados referentes a esta edição, correspondendo aos mesmos parâmetros analisados nas Tabelas 1 e 2 , respectivamente, da edição anterior.

Tabela 5 - Número de Game Jams já participadas anteriormente à IV A\&B

\begin{tabular}{|c|c|c|c|c|c|}
\hline Número de Game Jams & 0 & 1 & 2 & 3 & 4 \\
\hline Total de pessoas & 25 & 14 & 2 & 3 & 1 \\
\hline Participou exclusivamente da A\&B & - & 13 & 2 & - & - \\
\hline
\end{tabular}


Na Tabela 5 é possível verificar que cerca de $56 \%$ dos inscritos nunca havia participado de uma Game Jam previamente. Em relação aos $79 \%$ da terceira edição, notase uma queda relativa de $29 \%$ em relação à edição anterior, o que pode ser atribuído a um número menor de inscritos nesta edição. Aqui, a porcentagem de inscritos que não participou de nenhuma Game Jam exceto a A\&B foi de $89 \%$, percentual muito próximo ao da terceira edição.

Todavia, 33\% dos inscritos desta edição participou exclusivamente de outras edições da $A \& B$ contra cerca de $13 \%$ da edição anterior, representando um aumento relativo de $254 \%$. Tal aumento corrobora o comentário de um dos participantes em seu feedback do evento, que disse que "está se tornando uma tradição pra mim(sic) participar da $A \& B^{\prime \prime}$.

A redução no número de inscritos nesta edição pode ser atribuída, principalmente, à impossibilidade de divulgação de modo presencial nas instituições de ensino superior da região. Como visto na Tabela 6 , apenas 3 inscritos descobriram o evento pela divulgação em sua universidade - a qual nessa edição foi realizada exclusivamente por email. Em relação à edição III, isto representa uma queda de $85 \%$.

Além disso, na IV A\&B Game Jam, 24 inscritos descobriram o evento por meios diferentes de "Amigos" e "Faculdade". Tal número é ligeiramente superior ao da terceira edição, que, conforme Tabela 2, foi de 23 inscritos.

Tabela 6 - Número de inscritos que descobriram o evento por cada meio de divulgação utilizado na IV A\&B

\begin{tabular}{|l|l|l|l|l|l|}
\hline \multicolumn{7}{|c|}{ Como descobriu o evento } \\
\hline Amigos & Faculdade & Organização & WhatsApp & Site & Outro \\
\hline 18 & 3 & 9 & 11 & 3 & 1 \\
\hline
\end{tabular}

Sabendo que, após entrar em contato com o evento, muitos participantes convidam seus amigos e criam equipes, deduz-se que mesmo a queda de $45 \%$ no número de inscritos que foram convidados por amigos pode ser atribuída à falta de divulgação presencial nas universidades locais. Destaca-se, assim, a grande eficácia desse tipo de divulgação para atrair participantes ao evento.

A queda de $41 \%$ no número total de inscritos foi inferior à queda de $73 \%$ esperada pela organização do evento, tendo o número de inscritos surpreendido os organizadores de forma positiva.

Assim como na edição anterior, após a IV A\&B Game Jam um formulário de feedback foi enviado aos participantes, indagando-os sobre as milestones, a realização do evento de modo online, o trabalho da organização e sobre o evento como um todo. $\mathrm{O}$ resumo de algumas das questões do formulário é apresentado nas Tabela 7 e 8 . A partir destas respostas, percebe-se que, mesmo realizando a IV A\&B Game Jam em meio à pandemia, o evento foi um sucesso. 
Tabela 7 - Respostas para a pergunta sobre a realização online da IV A\&B

\begin{tabular}{|c|c|}
\hline \multicolumn{2}{|c|}{ Tema da pergunta: Realização da $A \& B$ online } \\
\hline $\begin{array}{l}\text { Aspectos comumente citados e opiniões } \\
\text { recorrentes }\end{array}$ & $\begin{array}{l}\text { Número de respostas } \\
\text { (percentual das respostas) }\end{array}$ \\
\hline Prefiro presencial & $7(23 \%)$ \\
\hline Prefiro virtual & $5(17 \%)$ \\
\hline Gosto tanto de presencial quanto virtual & $4(13 \%)$ \\
\hline \multicolumn{2}{|l|}{ Comentários de destaque: } \\
\hline \multicolumn{2}{|c|}{ "Em casa com a família, toda hora alguém atrapalhava com alguma coisa" } \\
\hline \multicolumn{2}{|c|}{$\begin{array}{l}\text { "Não consigo me dedicar } 100 \% \text { do tempo para a Jam e por isso uma Jam virtual é o que faz } \\
\text { com que eu consiga participar dela" }\end{array}$} \\
\hline $\begin{array}{l}\text { "O modelo presencial, como ocorreu ano pa } \\
\text { com os organizadores, teve os eventos de co }\end{array}$ & $\begin{array}{l}\text { eu um contato direto com a equipe e } \\
\text { também que foram bem divertidos" }\end{array}$ \\
\hline
\end{tabular}

Tabela 8 - Respostas para a pergunta sobre comentários gerais da IV A\&B

\begin{tabular}{|c|c|}
\hline \multicolumn{2}{|c|}{ Tema da pergunta: Comentários gerais sobre a IV A\&B Game Jam } \\
\hline $\begin{array}{l}\text { Aspectos comumente citados e opiniões } \\
\text { recorrentes }\end{array}$ & $\begin{array}{l}\text { Número de respostas } \\
\text { (percentual das respostas) }\end{array}$ \\
\hline Muito boa & $17(57 \%)$ \\
\hline Pretendo participar das próximas & $6(20 \%)$ \\
\hline \multicolumn{2}{|l|}{ Comentários de destaque: } \\
\hline \multicolumn{2}{|c|}{$\begin{array}{l}\text { "Foi uma experiência maravilhosa, me tirou da rotina de quarentena e consequentemente me } \\
\text { senti muito, mas muito melhor!" }\end{array}$} \\
\hline \multicolumn{2}{|l|}{ "Foi a coisa mais divertida dessa quarentena." } \\
\hline \multicolumn{2}{|c|}{$\begin{array}{l}\text { "O modelo presencial, como ocorreu ano passado, ofereceu um contato direto com a equipe } \\
\text { com os organizadores, teve os eventos de confraternização também que foram bem divertidos" }\end{array}$} \\
\hline $\begin{array}{l}\text { "Acho legal por ser um evento local, é mais } \\
\text { (esse é um motivo pelo qual não me sinto tã }\end{array}$ & $\begin{array}{l}\text { teragir com pessoal da cidade/região } \\
\text { articipando de outras jams online)" }\end{array}$ \\
\hline
\end{tabular}

Apesar do número reduzido de participantes, 11 grupos foram formados, um número superior ao da III $\mathrm{A} \& \mathrm{~B}$, ainda que 3 destes fossem compostos por apenas 1 
integrante. A média de integrantes por grupo na quarta edição foi de 3,2, inferior ao número de 4,9 da terceira edição.

Assim como foi realizado após a terceira edição, posteriormente à IV A\&B Game Jam o grupo PETComp realizou um feedback durante uma das reuniões semanais internas do grupo. Em tal feedback, destacou-se a facilidade de se organizar uma Game Jam virtual, mas que as maratonas presenciais aproximam muito mais os participantes e a organização, sendo muito mais proveitosas. Tendo em vista o engajamento apresentado pelos participantes no uso da plataforma Discord, sugeriu-se que ela seja usada numa edição híbrida, com atividades presenciais e online. Apesar das vantagens de realizar uma edição virtual, considerou-se que edições presenciais cumprem melhor a proposta da $\mathrm{A} \& \mathrm{~B}$ de estimular o cenário de desenvolvimento de jogos na região de Cascavel, sendo edições virtuais mais indicadas para uma abrangência geográfica maior. Este foco local da A\&B vai ao encontro do último comentário apresentado na Tabela 8.

\section{Conclusão}

A partir da comparação entre as estatísticas de inscritos da IV A\&B Game Jam com a III A\&B Game Jam nota-se que o projeto A\&B Game Jam tem atingido seus principais objetivos de estimular o cenário de desenvolvimento de jogos em Cascavel, sempre atraindo participantes para desenvolver seu primeiro jogo e se tornando uma tradição para todos os envolvidos.

Referente ao grupo PETComp, nota-se grande aprendizado dos membros do grupo na organização do evento, exigindo organização do grupo para a execução de todas as etapas e atividades requeridas e contato constante com a comunidade externa à universidade. Devido a isso, o projeto é atualmente a atividade de extensão realizada pelo grupo que atrai o maior número de participantes externos à Universidade, sendo apreciada por todos os membros envolvidos e crescendo a cada edição.

Apesar disso, a cada edição da A\&B Game Jam, novos desafios e dificuldades são enfrentados. Na III A\&B, como relatado previamente, houve grandes dificuldades com limitações da estrutura física utilizada. Durante o evento, a organização buscou manter diálogo constante, positivo e franco com os participantes a fim de amenizar tais limitações e impedir que elas comprometessem o evento para eles. Já na IV A\&B, a necessidade de se realizar a divulgação de modo exclusivamente online se mostrou a maior dificuldade, a qual resultou num número menor de participantes. Para ambos os casos, valiosas lições foram adquiridas, de modo que na próxima edição presencial a organização não medirá esforços para encontrar um local mais apropriado para a maratona, enquanto a divulgação online ocorrerá de modo mais intenso e com maior prazo.

\section{Agradecimentos}

Ao MEC-SESU, pelo financiamento deste projeto de extensão a partir do Programa de Educação Tutorial (PET), aos petianos egressos Angelo José Orssatto, Danielly Omori Antunes de Oliveira, Gabriela Paola Sereniski, Luani Regina Ebert, Lucas Frank Hollman, Valquíria Prestes Belusso, a todos os participantes da III e IV A\&B Game Jams, por tornar este evento possível, à Gracom - School of Visual Effects, Player One e Copex, pelo patrocínio da terceira edição do evento, a Alonso Lucca Fritz e Igor Arnaldo de Alencar por compartilharem seus conhecimentos através dos minicursos e palestras 
realizados e a Javan Bocchi, por sua inestimável colaboração na criação da A\&B Game Jam.

\section{Referências}

Kultima, A. (2015) "Defining Game Jam", Proceedings of the 10th International Conference on the Foundations of Digital Games (FDG 2015), Pacific Grove, CA, USA.

Locke, R. et al. (2015) "The Game Jam Movement: Disruption, Performance and Artwork". Proceedings of the 10th International Conference on the Foundations of Digital Games (FDG 2015), Pacific Grove, CA, USA.

Reng, L. et al. (2013) "The Motivational Power of Game Communities - Engaged through Game Jamming". Proceedings of the 8th International Conference on the Foundations of Digital Games (FDG 2013), Crete, Chania, Greece.

LPE (2019) “The Video Games' Industry is Bigger Than Hollywood". LPE Esports, https://lpesports.com/e-sports-news/the-video-games-industry-is-bigger-thanhollywood, Outubro.

Takahashi, D. (2017) "The U.S. game industry has 2,457 companies supporting 220,000 jobs". VentureBeat, , https://venturebeat.com/2017/02/14/the-u-s-game-industry-has2457-companies-supporting-220000-jobs/, Outubro.

Pereira, E. (2020) "Ednaldo Pereira", https://twitter.com/oednaldopereira, Outubro.

$\begin{array}{lccccc}\text { Petcomp } \quad \text { (2019) } & \text { "III } & \text { A\&B } & \text { Game } & \text { Jam". } & \text { GameJolt, } \\ \text { http://jams.gamejolt.io/abgamejam3, Outubro. } & & & \\ \text { Petcomp (2020) "IV } & \text { A\&B } & \text { Game } & \text { Jam". } & \text { GameJolt, } \\ \text { http://jams.gamejolt.io/abgamejam4, Outubro. } & & & \end{array}$

